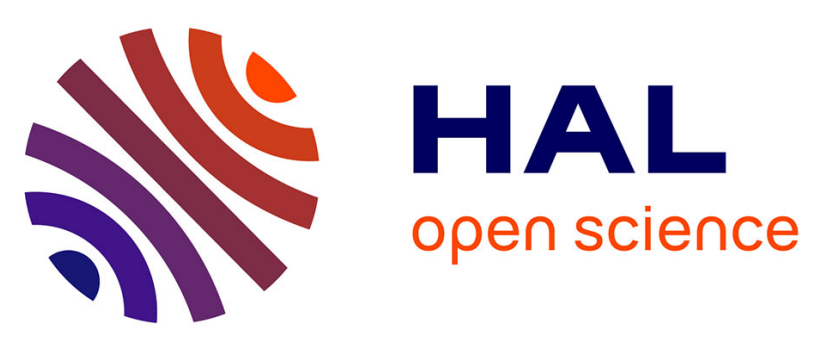

\title{
On the Combined Effect of Pressure and Third Invariant on Yielding of Porous Solids With von Mises Matrix
}

Oana Cazacu, Benoît Revil-Baudard, Ricardo A. Lebensohn, Mihail Garajeu

\section{To cite this version:}

Oana Cazacu, Benoît Revil-Baudard, Ricardo A. Lebensohn, Mihail Garajeu. On the Combined Effect of Pressure and Third Invariant on Yielding of Porous Solids With von Mises Matrix. Journal of Applied Mechanics, 2013, 80 (6), 10.1115/1.4024074 . hal-01656806

\section{HAL Id: hal-01656806 https://hal.science/hal-01656806}

Submitted on 6 Dec 2017

HAL is a multi-disciplinary open access archive for the deposit and dissemination of scientific research documents, whether they are published or not. The documents may come from teaching and research institutions in France or abroad, or from public or private research centers.
L'archive ouverte pluridisciplinaire HAL, est destinée au dépôt et à la diffusion de documents scientifiques de niveau recherche, publiés ou non, émanant des établissements d'enseignement et de recherche français ou étrangers, des laboratoires publics ou privés. 


\title{
On the combined effect of pressure and third invariant on yielding of porous solids with von Mises matrix
}

\author{
Oana Cazacu $^{1 *}$, Benoit Revil-Baudard ${ }^{1}$, Ricardo A. Lebensohnn ${ }^{2}$ and Mihail Gărăjeu ${ }^{3}$ \\ ${ }^{1}$ Department of Mechanical and Aerospace Engineering, University of Florida, \\ REEF, 1350 N. Poquito Rd, Shalimar, FL 32579, USA. \\ ${ }^{2}$ Los Alamos National Laboratory, MS G755, Los Alamos, NM 87545, USA. \\ ${ }^{3}$ Université Aix-Marseille, CNRS, M2P2 UMR7340, 13397 Marseille, France.
}

\begin{abstract}
In this paper, a new plastic potential for porous solids with von Mises perfectly-plastic matrix containing spherical cavities is derived using a rigorous limit analysis approach. For stress-triaxialities different from 0 and $\pm \infty$, the dilatational response depends on the signs of the mean stress and the third invariant of the stress deviator. The classic Gurson potential is an upper-bound of the new criterion. A full-field dilatational viscoplastic Fast Fourier Transform (FFT)-based approach is also used to generate numerical gauge surfaces for the porous material. The numerical calculations confirm the new features of the dilatational response, namely: a very specific dependence with the signs of the mean stress and the third invariant that results in a lack of symmetry of the yield surface.
\end{abstract}

Keywords: porous ductile solids; coupled mean stress third-invariant effect; tensioncompression asymmetry; limit analysis.

Corresponding author: Tel: +1 850833 9350; fax: +1 8508339366 .

E-mail address: cazacu@reef.ufl.edu 


\section{Introduction}

The most widely used yield criterion for isotropic porous solids containing randomly distributed spherical voids was developed by Gurson [1]. The derivation was made using limit analysis, the matrix material being considered rigid-perfectly plastic and obeying von Mises yield criterion. The expression of Gurson's yield criterion is:

$\Phi=\left(\frac{\Sigma_{\mathrm{e}}}{\sigma_{\mathrm{T}}}\right)^{2}+2 \mathrm{f} \cosh \left(\frac{3 \Sigma_{\mathrm{m}}}{2 \sigma_{\mathrm{T}}}\right)-1-\mathrm{f}^{2}=0$

where $f$ is the porosity, $\Sigma_{\mathrm{e}}$ is the von Mises effective stress, $\Sigma_{\mathrm{m}}$ is the mean stress, and $\sigma_{\mathrm{T}}$ is the tensile yield stress of the fully-dense material. Based on results of 2-D axisymmetric finite-element (FE) studies additional fitting parameters, $\mathrm{q}_{1}, \mathrm{q}_{2}, \mathrm{q}_{3}=\mathrm{q}_{1}^{2}$ were introduced in the expression (1) Gurson's criterion by Tvergaard [2] (see also Tvergaard and Needleman [3]). Modifications of the Gurson criterion based on the use of more sophisticated trial velocity fields, e.g. derived from the exact Eshelby solution for the ellipsoidal inclusion problem in an infinite elastic matrix have also been proposed (see Monchiet et al. [4]). It is worth noting that Gurson's original criterion [1] and all the Gurson-like criteria [3-4] display symmetry with respect to both the axis $\left(\Sigma_{\mathrm{m}}=0\right)$ and the axis $\left(\Sigma_{\mathrm{e}}=0\right)$, i.e. it is invariant with respect to both transformations $\left(\Sigma_{\mathrm{m}}, \Sigma^{\prime}\right) \rightarrow\left(\Sigma_{\mathrm{m}},-\Sigma^{\prime}\right)$ and $\left.\left(\Sigma_{\mathrm{m}}, \Sigma^{\prime}\right) \rightarrow\left(-\Sigma_{\mathrm{m}}, \Sigma^{\prime}\right)\right)$. It is generally accepted that these strong symmetry properties of the yield surface and the insensitivity to the third invariant could have not been predicted a priori in the context of limit analysis (see Leblond [5]). Yet, the exact plastic potential of an isotropic porous solid with von Mises matrix should be an even function in stress (Duva and Hutchinson [6]), so it should be invariant only to the transformation $\left(\Sigma_{\mathrm{m}}, \Sigma^{\prime}\right) \rightarrow\left(-\Sigma_{\mathrm{m}},-\Sigma^{\prime}\right)$

Constitutive potentials for porous solids with incompressible power-law matrix containing a dilute concentration of spherical voids or penny-shaped cracks were derived by Duva and Hutchinson [6]. The theory also applies to rate-independent perfect plasticity (in the limit when the power law exponent tends to infinity). Based on the 
concept of "linear comparison composite", Ponte Castaneda [7] and later Kaisalam and Ponte Castaneda [8] developed rigorous upper-bounds of the overall potential of plastic and viscoplastic solids containing randomly oriented ellipsoidal cavities.

All the above potentials for porous materials depend only on the mean stress $\Sigma_{\mathrm{m}}$ and the von Mises effective stress $\Sigma_{\mathrm{e}}$. However, FE cell calculations for axisymmetric or general 3-D tensile loading configurations have shown that a given value of the stress-triaxiality does not define uniquely the yield point and a slight dependence on the third invariant of the stress deviator $\mathrm{J}_{3}^{\Sigma}=\frac{1}{3} \operatorname{tr}\left(\boldsymbol{\Sigma}^{\prime 3}\right)$. In the plane $\left(\Sigma_{\mathrm{m}}, \Sigma_{\mathrm{e}}\right)$, the yield points corresponding to $\mathbf{J}_{3}^{\Sigma} \geq 0$ (axial stress greater than the lateral stress) are below that corresponding to $\mathbf{J}_{3}^{\Sigma} \leq 0$ (axial stress less than lateral stress) (e.g. Richelsen and Tvergaard [9], Kim et al. [10], McElwain et al. [11]). Within the framework of non-linear homogenization, theoretical results by Ponte Castaneda and Suquet [12] for weakly contrasted materials, and by Danas et al. [13] in the context of the "second-order" method of Ponte Castaneda [14] adapted to the case of porous materials with von Mises matrix, also show dependence with the third invariant of the stress deviator. Further evidence of third invariant effects was also provided by Julien et al. [15] who performed periodic unit cell calculations for both tensile and compressive stress states using a Fast Fourier Transform (FFT)-based spectral formulation [16,17]. Moreover, Cazacu and Stewart [18] conducted axisymmetric FE cell calculations for both tensile and compressive stress states. In the plane $\left(\Sigma_{\mathrm{m}}, \Sigma_{\mathrm{e}}\right)$, their results describe two yield curves. For tensile loadings, the yield curve corresponding to $\mathbf{J}_{3}^{\Sigma} \geq 0$ (axial stress greater than the lateral stress) is below that corresponding to $\mathrm{J}_{3}^{\Sigma} \leq 0$ (axial stress less than lateral stress), while for compressive loadings the reverse occurs. Very recently, Lebensohn and Cazacu [19] reported numerical results, obtained with Lebensohn et al.'s dilatational viscoplastic implementation [20] of the FFT-based formulation for porous viscoplastic fcc polycrystals with random texture and deforming by slip at single crystal level. A very specific dependence of the gauge surfaces with the signs of both the stress-triaxiality $\mathrm{X}_{\sigma}=\frac{\Sigma_{\mathrm{m}}}{\Sigma_{\mathrm{e}}}$ and the third invariant was revealed. Specifically, it was shown that a point of 
the gauge surface for $J_{3}^{\Sigma} \geq 0$ corresponding to a given stress-triaxiality $X_{\sigma}$ is symmetric with respect to the axis $\left(\Sigma_{\mathrm{m}}=0\right)$ to the point on the other gauge surface $\left(\mathrm{J}_{3}^{\Sigma} \leq 0\right)$ corresponding to $-\mathrm{X}_{\sigma}$. Given that in these calculations the matrix was a heterogeneous polycrystalline aggregate, this specific dependence needs to be verified for porous materials with homogenous von Mises matrix. Such evidence will be provided in this paper. The main conclusion from the aforementioned numerical studies is that the yield behavior of porous materials with von Mises matrix should depend on the combined effects of the second and third invariant of the stress deviator (Lode parameter dependence). Along these lines, phenomenological criteria for porous solids involving sensitivity to the Lode parameter have been proposed (e.g. Bao and Wierzbicki [21]). An approximate plastic potential that involves the third invariant of the stress deviator was derived by Cazacu and Stewart [18] in the framework of limit analysis. Yet, in this formulation, the isotropic matrix is governed by the pressure-insensitive yield criterion of Cazacu et al. [22] that is an odd function in stress and as such displays strengthdifferential effects. Thus, the third-invariant sensitivity of the dilatational response of the voided polycrystal predicted by the criterion is due to the tension-compression asymmetry of the plastic flow of the matrix. If the plastic flow in the matrix does not display tension-compression asymmetry, the criterion reduces to Gurson [1] (for more details, see [18]).

In this paper, we demonstrate that the insensitivity of Gurson's criterion [1] to the third invariant and the ensuing strong symmetry properties of the yield surface are direct consequences of a simplifying hypothesis. Specifically, it is shown that this hypothesis results in neglecting combined effects of the mean stress and stress deviator on yielding of the porous solid. Furthermore, a new analytic yield criterion for a porous solid with matrix obeying the von Mises yield criterion is derived using a rigorous limit-analysis approach (Section 2). This criterion predicts a combined effect of the third invariant and mean stress. It is worth noting that this very specific dependence is demonstrated and not postulated. Moreover, the full-field dilatational viscoplastic FFT-based approach of Lebensohn et al. [23] is used to generate numerical gauge surfaces (Section 3). These numerical results also reveal the same coupling between the mean stress and third 
invariant, which induces a lack of symmetry of the yield surface with respect to both the axis $\Sigma_{\mathrm{e}}=0$ and $\Sigma_{\mathrm{m}}=0$ for stress-triaxialities different from 0 and $\pm \infty$.

\section{Derivation of the analytic yield criterion}

We shall now analytically derive an approximate plastic potential for porous materials with isotropic rigid-plastic matrix described by the von Mises criterion containing randomly distributed spherical voids. For this purpose, as in Gurson [1], we conduct a limit analysis of a hollow sphere using Rice and Tracey [24] trial velocity field. Let the

inner radius of the hollow sphere be denoted by $a$, and its outer radius $b=a f^{-1 / 3}$. The outer boundary $(r=b)$ is subjected to axisymmetric loading such that:

$\boldsymbol{\Sigma}=\Sigma_{11}\left(\mathbf{e}_{1} \otimes \mathbf{e}_{1}^{+} \mathbf{e}_{2} \otimes \mathbf{e}_{2}\right)+\Sigma_{33}\left(\mathbf{e}_{3} \otimes \mathbf{e}_{3}\right)$,

where $\boldsymbol{\Sigma}$ denotes the average stress field, while the inner boundary is considered to be traction-free. In the above equation, $\left(\mathbf{e}_{1}, \mathbf{e}_{2}, \mathbf{e}_{3}\right)$ are the unit vectors of a Cartesian coordinate system. Rice and Tracey [24] velocity field is given by $\mathbf{v}=\frac{\mathrm{b}^{3}}{\mathrm{r}^{2}} \mathrm{D}_{\mathrm{m}} \mathbf{e}_{\mathrm{r}}+\mathbf{D}^{\prime} \mathbf{x}$

where: $D_{m}=\frac{1}{3} \operatorname{tr} \mathbf{D}$, and $\mathbf{D}^{\prime}$ is the deviator of the macroscopic strain rate tensor, assumed to be constant. In Eq. (3) both Cartesian and spherical coordinates have been used. The principal values (unordered) of the local strain rate field $\mathbf{d}=\frac{1}{2}\left(\nabla \mathbf{v}+\nabla \mathbf{v}^{T}\right)$ corresponding to the velocity field $\mathbf{v}$ given by Eq. (3), are as follows: 


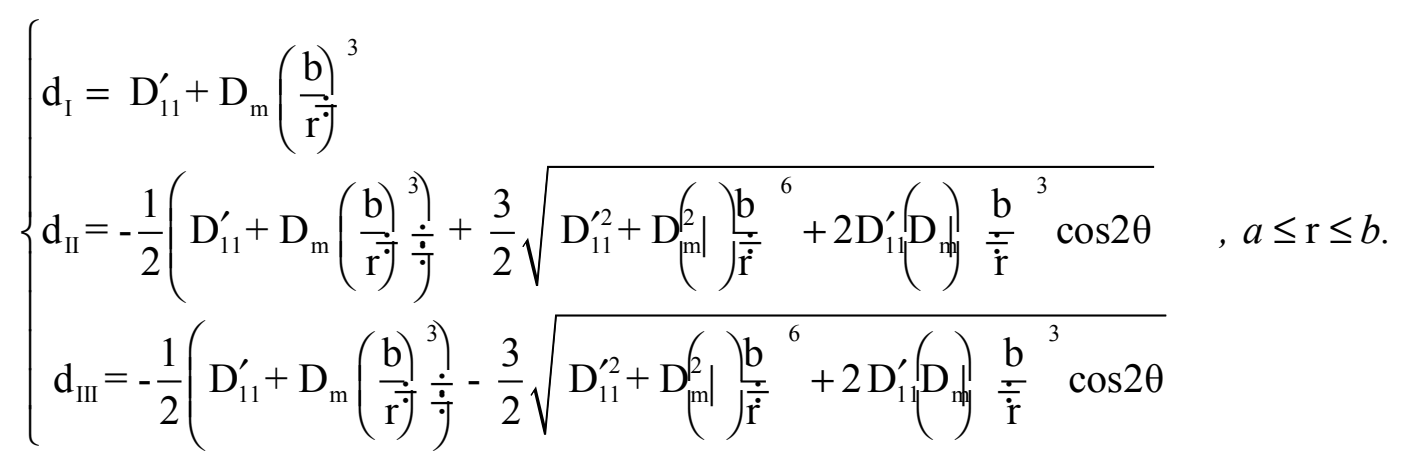

Using the associated flow rule, the local plastic dissipation corresponding to Von Mises criterion and the local strain rate field $\mathbf{d}$ is:

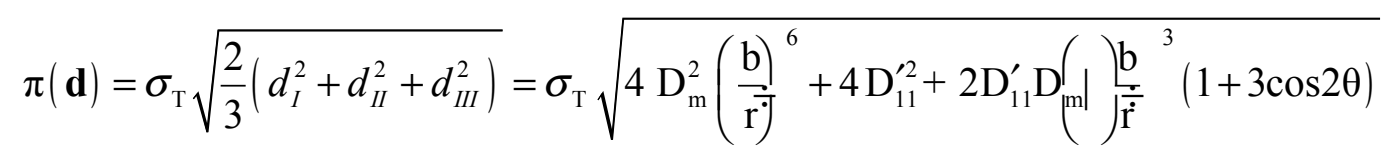

Since the trial velocity field $\mathbf{v}$ is incompressible and compatible with homogeneous strain rate boundary conditions (see Eq. (3)), Hill-Mandel ([25, 26]) lemma applies. Thus, an upper-bound estimate of the overall plastic potential of porous solid is provided by $\Pi^{+}(\mathbf{D})$, the average value over the hollow sphere of the local plastic dissipation $\pi(\mathbf{d})$, corresponding to this trial velocity field. Its expression is:

$$
\Pi^{+}(\mathbf{D})=\frac{1}{\mathrm{~V}} \int_{\Omega} \pi(\mathbf{d}) \mathrm{d} \Omega=\frac{\sigma_{\mathrm{T}}}{\mathrm{V}} \int_{\Omega} \sqrt{4 \mathrm{D}_{\mathrm{m}}^{2}(\mathrm{~b} / \mathrm{r})^{6}+4 \mathrm{D}_{11}^{\prime 2}+2 \mathrm{D}_{11}^{\prime} \mathrm{D}_{\mathrm{m}}(\mathrm{b} / \mathrm{r})^{3}(1+3 \cos 2 \theta)} \mathrm{d} \Omega
$$

with $\mathrm{V}=4 \pi \mathrm{b}^{3} / 3$ and $\Omega$ is the domain occupied by the matrix.

Remark: It is important to note that in Gurson [1], the following simplifying hypothesis was introduced: 
H1) In the expression (4) of the local plastic dissipation $\pi(\mathbf{d})$, the "crossed term" involving $\mathrm{D}_{\mathrm{m}} \mathrm{D}_{11}^{\prime}$ can be neglected .

Using this simplifying hypothesis, Gurson [1] proposed the following approximate plastic potential of the porous aggregate:

$\Pi^{++}(\mathbf{D})=\frac{1}{\mathrm{~V}} \int_{\Omega} \sqrt{4 \mathrm{D}_{\mathrm{m}}^{2}\left(\frac{\mathrm{b}}{\left.\mathrm{r}^{\mathrm{r}}\right)^{6}}+4 \mathrm{D}_{11}^{\prime 2}\right.} \mathrm{dV}$

and calculated the macroscopic stresses associated with $\Pi^{++}(\mathbf{D})$ as:

$\Sigma_{11}=\Sigma_{22}=\frac{\partial \Pi^{++}}{\partial \mathrm{D}_{11}}$ and $\Sigma_{33}=\frac{\partial \Pi^{++}}{\partial \mathrm{D}_{33}}$

or:

$\frac{\Sigma_{\mathrm{m}}}{\sigma_{T}}=\frac{1}{3} \frac{\partial \Pi^{++}}{\partial D_{m}}=\frac{2}{3} \ln \left(\frac{u+\sqrt{u^{2}+f^{2}}}{u+\sqrt{u^{2}+1}} \times \underset{f}{1} \stackrel{\dot{\dot{\dot{\rho}}}}{)}\right.$,

$\frac{\Sigma_{\mathrm{e}}}{\sigma_{T}}=\left|\frac{\partial \Pi^{++}}{\partial D_{e}}\right|=\sqrt{1+u^{2}}-\sqrt{u^{2}+f^{2}}$

In the above equations, $\mathrm{D}_{\mathrm{e}}=\sqrt{\frac{2}{3} \mathrm{D}_{\mathrm{ij}}^{\prime} \mathrm{D}_{\mathrm{ij}}^{\prime}}=2\left|\mathrm{D}_{11}^{\prime}\right|$ is the macroscopic equivalent strain rate while $u \stackrel{\text { def }}{=} \frac{2\left|D_{m}\right|}{D_{e}}$ is the absolute value of the strain-triaxiality given by $u=\frac{\operatorname{def}}{=} \frac{2\left|D_{m}\right|}{D_{e}}$; $\Sigma_{\mathrm{m}}=\frac{1}{3} \operatorname{tr}(\boldsymbol{\Sigma})$ and $\Sigma_{\mathrm{e}}=\sqrt{\frac{3}{2} \Sigma_{i j}^{\prime} \Sigma_{i j}^{\prime}}$ are the mean macroscopic stress and effective macroscopic stress, respectively. Equations (8) define Gurson's [1] yield locus in a 
parametric form, which can be further cast in the classical form, given by Eq. (1), by eliminating the parameter $u$ (for details, see [27]).

In what follows, we show that hypothesis H1) is too restrictive (see also [28]). Specifically, we demonstrate that the insensitivity of Gurson's criterion to the third invariant and the ensuing strong symmetry properties of the Gurson's yield surface with respect to both the axis $\left(\Sigma_{\mathrm{e}}=0\right)$ and the axis $\left(\Sigma_{\mathrm{m}}=0\right)$ are a direct consequence of this hypothesis. Furthermore, we obtain explicit expressions for the plastic potential $\Pi^{+}(\mathbf{D})$ and provide an approximate yield criterion for the porous aggregate in the form:

$$
\left\{\begin{array}{l}
\Sigma_{m}=\frac{1}{3} \frac{\partial \Pi^{+}}{\partial \mathrm{D}_{m}} \\
\Sigma_{\mathrm{e}}=\left|\frac{\partial \Pi^{+}}{\partial \mathrm{D}_{\mathrm{e}}}\right|
\end{array}\right.
$$

Furthermore, since $\Pi^{+}(\mathbf{D}) \leq \Pi^{++}(\mathbf{D})$, Gurson's criterion is an upper bound of the new approximate criterion (9). Moreover, the new criterion has the following remarkable properties:

(i) Yielding depends on the sign of the third invariant of the stress deviator.

(ii) For stress-triaxialities $\mathrm{X}_{\sigma}=\frac{\Sigma_{\mathrm{m}}}{\Sigma_{\mathrm{e}}}$ different from 0 and $\pm \infty$, yielding depends on the signs of $J_{3}^{\Sigma}$ and the mean stress. Specifically, an yield point characterized by $J_{3}^{\Sigma} \geq 0$ and a given stress-triaxiality $\mathrm{X}_{\sigma}$ is symmetric, with respect to the axis $\Sigma_{\mathrm{m}}=0$, to the yield point characterized by $\mathrm{J}_{3}^{\Sigma} \leq 0$ and $-\mathrm{X}_{\sigma}$. 
(iii) Irrespective of the sign of the third invariant of the stress deviator, for purely deviatoric loading, yielding occurs at $\Sigma_{\mathrm{e}}=\sigma_{T}(1-f)$, while for purely hydrostatic loadings, yielding occurs at: $\left|\Sigma_{\mathrm{m}}\right|=\frac{2}{3} \sigma_{T} \ln f$.

\section{Proof:}

For $\mathrm{D}_{11}^{\prime}>0$ and $\mathrm{D}_{\mathrm{m}}=\frac{1}{3} \operatorname{tr} \mathbf{D} \geq 0$, the overall plastic dissipation is:

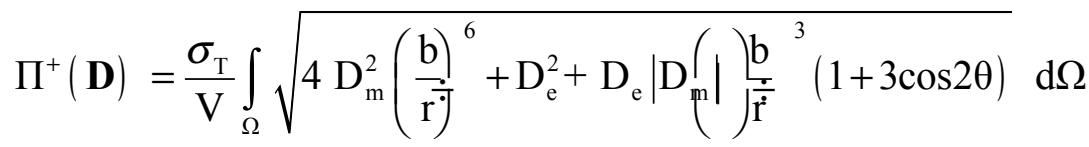

so $\frac{\partial \Pi^{+}}{\partial \mathrm{D}_{11}}>\frac{\partial \Pi^{+}}{\partial \mathrm{D}_{33}}$ and $\frac{\partial \Pi^{+}}{\partial \mathrm{D}_{m}}>0$. Hence, the stress state at yielding is such that $\Sigma_{11}>\Sigma_{33}$ so $\mathbf{J}_{3}^{\Sigma}<0$ and $\Sigma_{\mathrm{m}}>0$. On the other hand, for $\mathrm{D}_{11}^{\prime}<0$ and $\mathrm{D}_{\mathrm{m}}=\frac{1}{3} \operatorname{tr} \mathbf{D} \geq 0$ :

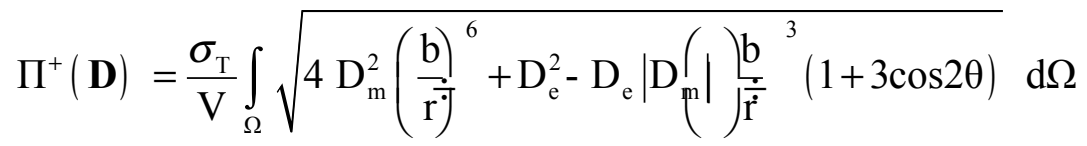

so $\frac{\partial \Pi^{+}}{\partial \mathrm{D}_{33}}>\frac{\partial \Pi^{+}}{\partial \mathrm{D}_{11}}$ and $\frac{\partial \Pi^{+}}{\partial \mathrm{D}_{m}}>0$ which corresponds to $\Sigma_{\mathrm{m}}>0$ and $\mathrm{J}_{3}^{\Sigma}>0$.

Moreover, the overall plastic dissipation $\Pi^{+}(\mathbf{D})$ is invariant under the transformation: $\left(D_{m}, D_{11}^{\prime}\right) \rightarrow\left(-D_{m},-D_{11}^{\prime}\right)$, so the expression of $\Pi^{+}(\mathbf{D})$ for stress states corresponding to ( $\Sigma_{\mathrm{m}} \geq 0$ and $\mathrm{J}_{3}^{\Sigma} \geq 0$ ) is identical with that corresponding to stress states for which ( $\Sigma_{\mathrm{m}} \leq 0$ and $\mathrm{J}_{3}^{\Sigma} \leq 0$ ). Thus, according to the new criterion (9) yielding depends on the sign of the third invariant of the stress deviator. Moreover, for stress-triaxialities different from 
0 and $\pm \infty$, (for which $\mathrm{D}_{\mathrm{e}} \mathrm{D}_{\mathrm{m}} \neq 0$ ), yielding also depends on the sign of the mean stress (which has the same sign as $\mathrm{D}_{\mathrm{m}}$ ). Furthermore, Eqs. (10) and (11) show that an yield point characterized by $\mathrm{J}_{3}^{\Sigma} \geq 0$ and a given stress-triaxiality $\mathrm{X}_{\boldsymbol{\sigma}}$ is symmetric, with respect to the axis $\Sigma_{\mathrm{m}}=0$, to the yield point characterized by $\mathrm{J}_{3}^{\Sigma} \leq 0$ and $-\mathrm{X}_{\boldsymbol{\sigma}}$. Obviously, for the special cases of purely hydrostatic $\left(\mathbf{D}^{\prime}=0\right)$, or purely deviatoric loading $\left(\mathrm{D}_{\mathrm{m}}=0\right)$, $\Pi^{+}(\mathbf{D})=\Pi^{++}(\mathbf{D})$, so the new criterion (9) coincides with Gurson [1].

In what follows, we provide the explicit parametric representation of the yield surface (9). The details of the calculations, i.e. substitution of Eqs. (10) and (11) into Eq. (9) and further integration, are given in Appendix 1.

The new approximate criterion for the porous aggregate is given by:

a) For $\mathrm{J}_{3}^{\Sigma} \leq 0, \Sigma_{\mathrm{m}} \geq 0$, and any value of $u=\frac{2\left|D_{m}\right|}{D_{e}}$ :

$$
\begin{aligned}
& \left(\frac{\Sigma_{m}}{\sigma_{T}}=\frac{1}{9} \frac{1-f}{u}+\frac{1}{3} \ln \left(\frac{u^{2}-u f+f^{2}}{u^{2}-u+1} \frac{1}{\left.f^{2} \cdot\right)}\right.\right.
\end{aligned}
$$

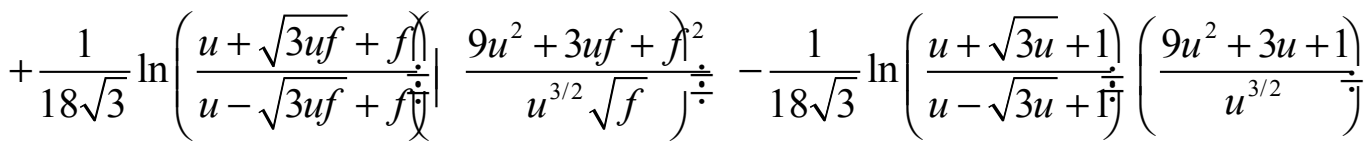

$$
\begin{aligned}
& +\frac{2}{18 \sqrt{3}}\left(\tan ^{-1}\left(2 \sqrt{\frac{u}{f}}+\sqrt{\frac{3}{\dot{⿰}}}\right)-\tan ^{-1} \mid 2 \sqrt{\frac{u}{f}}-\sqrt{\frac{3}{3}}\right) \frac{2}{\frac{\dot{T}}{j}}-\frac{2}{18 \sqrt{3}}\left(\tan ^{-1}(2 \sqrt{u}+\sqrt{3})-\tan ^{-1}(2 \sqrt{u}-\sqrt{3})\right) \\
& \frac{\Sigma_{e}}{\sigma_{T}}=\frac{1-f}{2}+\frac{1}{4 \sqrt{3 u}}\left[( u ^ { 2 } - u + 1 ) \operatorname { l n } \left(\frac{u+\sqrt{3 u}+1)}{u-\sqrt{3 u}+\dot{\dot{D}}}-\frac{\left(u^{2}-u f+f^{2}\right)}{\sqrt{f}} \ln \left(\frac{u+\sqrt{3 u f}+f)}{u-\sqrt{3 u f}+f \dot{\dot{\dot{j}}}}\right]\right.\right.
\end{aligned}
$$

b) For $\mathrm{J}_{3}^{\Sigma} \leq 0$ and $\Sigma_{\mathrm{m}} \leq 0$ :

b1) If $u \leq f$ : 


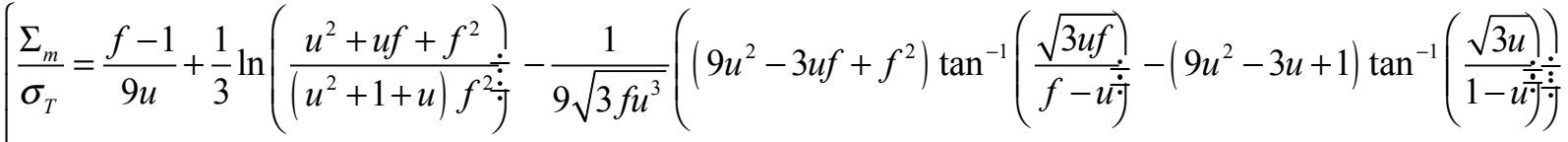

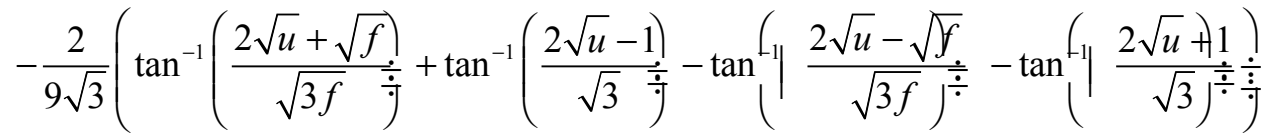

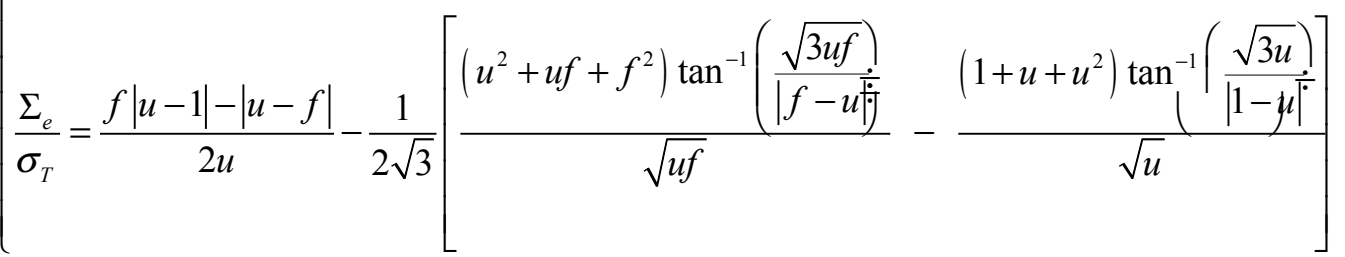

b2) If $f<u<1$ :

$$
\begin{aligned}
& \int \frac{\Sigma_{m}}{\sigma_{T}}=\frac{2}{3} \ln (3)-\frac{2}{18} \frac{\pi}{\sqrt{3}}-\frac{1+f-2 u}{9 u}-\frac{1}{3} \ln \left(\frac{\left.\left(u^{2}+1+u\right)\left(u^{2}+u f+f^{2}\right)\right)}{f^{2}} \underset{\dot{\vdots}}{\dot{j}}\right.
\end{aligned}
$$

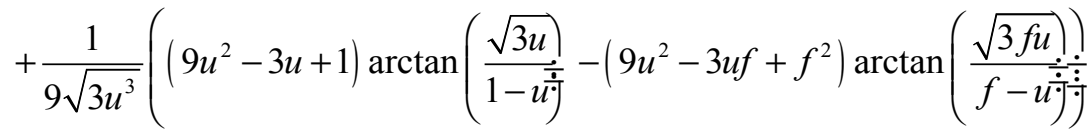

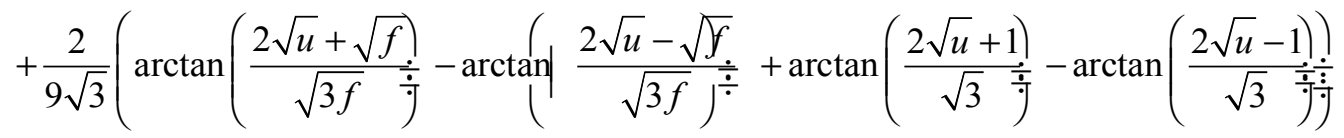

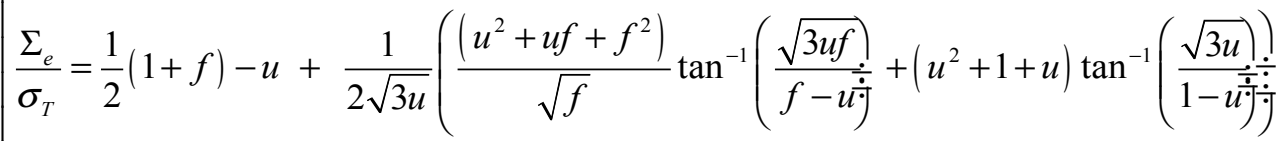

b3) If $u \geq 1$ : 


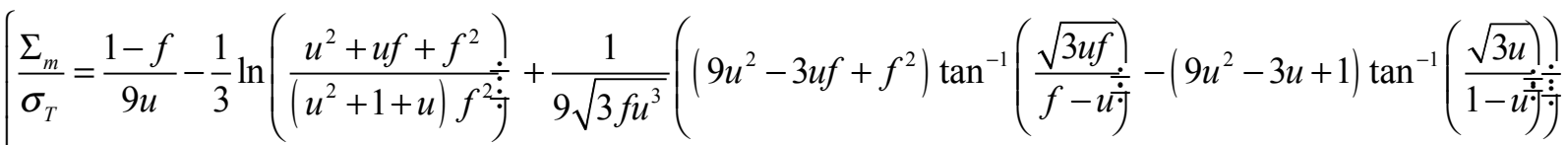

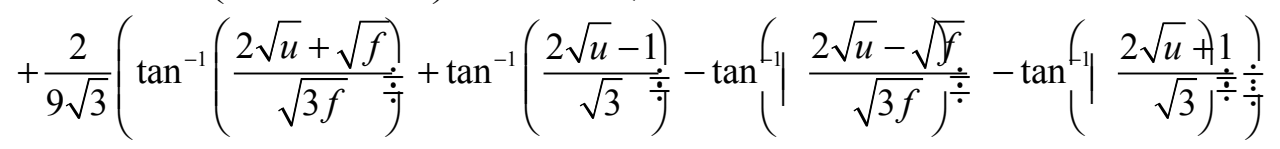

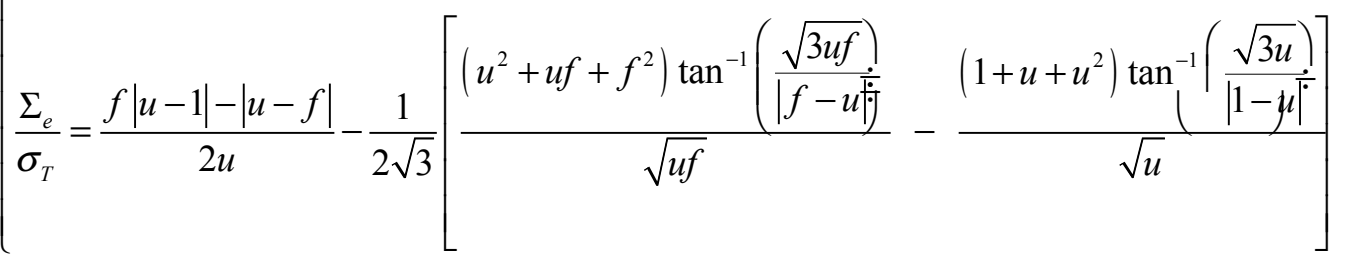

Based on the property (ii) demonstrated earlier, the parametric representation of the yield locus corresponding to $\mathrm{J}_{3}^{\Sigma} \geq 0$ can be easily obtained from Eqs. (12):

c) For $\mathrm{J}_{3}^{\Sigma} \geq 0$ and $\Sigma_{\mathrm{m}} \geq 0$ :

$$
\left\{\begin{array}{l}
\frac{\Sigma_{m}}{\sigma_{T}}=-\left.\frac{\Sigma_{m}}{\sigma_{T}}\right|_{\mathrm{J}_{3}^{\Sigma} \leq 0, \Sigma_{m} \leq 0} \\
\frac{\Sigma_{e}}{\sigma_{T}}=\left.\frac{\Sigma_{e}}{\sigma_{T}}\right|_{3} ^{\Sigma} \leq 0, \Sigma_{m} \leq 0
\end{array}\right.
$$

where the right-hand expressions are given by Eqs. (12b-d).

d) For $\mathrm{J}_{3}^{\Sigma} \geq 0$ and $\Sigma_{\mathrm{m}} \leq 0$ :

$$
\left\{\begin{array}{l}
\frac{\Sigma_{m}}{\sigma_{T}}=-\left.\frac{\Sigma_{m}}{\sigma_{T}}\right|_{\mathrm{J}_{3}^{\Sigma} \leq 0, \Sigma_{m} \geq 0} \\
\frac{\Sigma_{e}}{\sigma_{T}}=\left.\frac{\Sigma_{e}}{\sigma_{T}}\right|_{\mathrm{J}_{3}} \leq 0, \Sigma_{m} \geq 0
\end{array}\right.
$$


where the right-hand expressions are given by Eq. (12a).

It can be easily seen that for $u \rightarrow \infty$ (purely hydrostatic loading) in Eq. (12a), we obtain: $\left|\Sigma_{\mathrm{m}}\right|=\frac{2}{3} \sigma_{T} \ln f$, and $\Sigma_{\mathrm{e}}=0$; and for $u \rightarrow 0$ (purely deviatoric loading), $\Sigma_{\mathrm{m}}=0$, and $\Sigma_{\mathrm{e}}=\sigma_{T}(1-f)$, irrespective of the sign of the third invariant of the stress deviator. As already mentioned, this is to be expected since, for the respective stress states, the proposed criterion and Gurson [1] coincide.

For illustration of the features of the new criterion (Eq (12)), in what follows we present plots of the resulting yield surface in the plane $\left(\Sigma_{\mathrm{m}}, \Sigma_{\mathrm{e}}\right)$. For axisymmetric loading, $\boldsymbol{\Sigma}=\Sigma_{11}\left(\mathbf{e}_{1} \otimes \mathbf{e}_{1}+\mathbf{e}_{2} \otimes \mathbf{e}_{2}\right)+\Sigma_{33}\left(\mathbf{e}_{3} \otimes \mathbf{e}_{3}\right)$, von Mises equivalent stress is $\Sigma_{e}=\left|\Sigma_{11}-\Sigma_{33}\right|$, the mean stress is $\Sigma_{m}=\left(2 \Sigma_{11}+\Sigma_{33}\right) / 3$, and the third invariant of the stress deviator is $\mathbf{J}_{3}^{\Sigma}=-\frac{2}{27}\left(\Sigma_{11}-\Sigma_{33}\right)^{3}$, so $\mathbf{J}_{3}^{\Sigma} \leq 0$ corresponds to stress states for which $\Sigma_{11} \geq \Sigma_{33}$ while $\mathbf{J}_{3}^{\Sigma} \geq 0$ corresponds to stress states for which $\Sigma_{11} \leq \Sigma_{33}$.

Figure 1 shows the representation in the plane $\left(\Sigma_{\mathrm{m}}, \Sigma_{\mathrm{e}}\right)$ of the yield surface corresponding to $J_{3}^{\Sigma} \leq 0$ (Eqs. 12) for a porosity $\mathrm{f}=0.05$. Clearly, for triaxialities different from 0 and $\pm \infty$, the yield surface according to the new criterion is no longer symmetric with respect to either the axis $\Sigma_{\mathrm{m}}=0$ or $\Sigma_{\mathrm{e}}=0$. For a given absolute value of the strain-triaxiality $u$, there are two yield points on the surface with different absolute value of the stresstriaxiality. For example, in Fig. 1 are shown the yield points A $\left(\Sigma_{\mathrm{m}} / \sigma_{\mathrm{T}}=1.861, \Sigma_{\mathrm{e}} / \sigma_{\mathrm{T}}\right.$ $=0.400)$ and $\mathrm{B}\left(\Sigma_{\mathrm{m}} / \sigma_{\mathrm{T}}=-1.867, \Sigma_{\mathrm{e}} / \sigma_{\mathrm{T}}=0.381\right)$, which correspond to $\mathrm{u}=0.935$.

For the same porosity $f=0.05$, the yield surface corresponding to $\mathrm{J}_{3}^{\Sigma} \geq 0$ (stress states for which $\Sigma_{11} \leq \Sigma_{33}$ ) is plotted in Fig. 2. An yield point corresponding to a given stresstriaxiality $\mathrm{X}_{\sigma}$ is symmetric, with respect to the axis $\Sigma_{\mathrm{m}}=0$, to the point on the other yield 
surface $\left(\mathrm{J}_{3}^{\Sigma} \leq 0\right)$ corresponding to $-\mathrm{X}_{\sigma}$. For example, yield point $\mathrm{C}\left(\Sigma_{\mathrm{m}} / \sigma_{\mathrm{T}}=-1.861\right.$, $\Sigma_{\mathrm{e}} / \sigma_{\mathrm{T}}=0.400$ and $\mathrm{J}_{3}^{\Sigma} / \sigma_{\mathrm{T}}=0.004741$ ) has the same absolute value of stress-triaxiality as the yield point $\mathrm{A}\left(\Sigma_{\mathrm{m}} / \sigma_{\mathrm{T}}=1.861, \Sigma_{\mathrm{e}} / \sigma_{\mathrm{T}}=0.400\right.$ and $\left.\mathrm{J}_{3}^{\Sigma} / \sigma_{\mathrm{T}}=-0.004741\right)$ (see Fig. 1). In other words, the yield locus is invariant to transformations: $\left(\Sigma_{\mathrm{m}}, \Sigma_{\mathrm{e}}, \mathrm{J}_{3}^{\Sigma}\right) \rightarrow\left(-\Sigma_{\mathrm{m}}, \Sigma_{\mathrm{e}},-\mathrm{J}_{3}^{\Sigma}\right)$ or $\left(\Sigma_{\mathrm{m}}, \Sigma^{\prime}\right) \rightarrow\left(-\Sigma_{\mathrm{m}},-\Sigma^{\prime}\right)$

Figure 3 shows the yield curves according to the proposed criterion corresponding to $\mathrm{J}_{3}^{\Sigma} \geq 0$ and $\mathrm{J}_{3}^{\Sigma} \leq 0$, respectively, and Gurson yield surface, for the same porosity ( $\mathrm{f}=5 \%$ ). It is clearly seen that Gurson [1] is an upper-bound of the proposed criterion.

While Gurson [1] is invariant to both $\left(\Sigma_{\mathrm{m}}, \Sigma^{\prime}\right) \rightarrow\left(\Sigma_{\mathrm{m}},-\Sigma^{\prime}\right)$ and $\left.\left(\Sigma_{\mathrm{m}}, \Sigma^{\prime}\right) \rightarrow\left(-\Sigma_{\mathrm{m}}, \Sigma^{\prime}\right)\right)$ (i.e. it is symmetric to both the axis $\left(\Sigma_{\mathrm{e}}=0\right)$ and the axis $\left.\left(\Sigma_{\mathrm{m}}=0\right)\right)$, the new criterion is invariant only to $\left(\Sigma_{\mathrm{m}}, \Sigma^{\prime}\right) \rightarrow\left(-\Sigma_{\mathrm{m}},-\Sigma^{\prime}\right)$ and is insensitive to the sign of $\Sigma_{\mathrm{m}}$ only for purely deviatoric states $\left(\Sigma_{\mathrm{m}}=0\right)$. Note that according to the proposed criterion for $\Sigma_{\mathrm{m}} \geq 0$, the response is softer for $\mathrm{J}_{3}^{\Sigma} \geq 0$ than for $\mathrm{J}_{3}^{\Sigma} \leq 0$ (see also Fig. 4, showing different zooms on the yield surfaces in the tensile quadrant). For purely deviatoric loading, the response is the same, and the effect of $\mathrm{J}_{3}^{\Sigma}$ becomes noticeable with increasing triaxiality (see Figs. 4a-b). The normal to the yield surfaces are clearly different, determining distinct plastic flow directions and resulting damage evolution. For triaxialities approaching infinity, the effect of $\mathrm{J}_{3}^{\Sigma}$ starts to decrease, and both yield surfaces coincide at the purely hydrostatic point (see Fig. 4c).

For completion, Fig. 5 shows the yield surface in the plane $\left(\Sigma_{11}-\Sigma_{33}, \Sigma_{m}\right)$ according to the new criterion and Gurson [1], for several porosities, 1\%, 5\%, and 15\%. All yield surfaces show the same trends described above. Irrespective of the porosity, the intersection of the curves corresponding to $\left.\mathrm{J}_{3}^{\Sigma} \geq 0\left(\Sigma_{11} \leq \Sigma_{33}\right)\right)$ and $\mathrm{J}_{3}^{\Sigma} \leq 0\left(\Sigma_{11} \geq \Sigma_{33}\right)$ belong to the axis $\Sigma_{\mathrm{m}}=$ 
0 (i.e. the yield surface is continuous at $\Sigma_{\mathrm{m}}=0$ ); the proposed criterion coincides with Gurson [1] only for the purely hydrostatic $\left(\Sigma_{11}=\Sigma_{22}=\Sigma_{33}\right)$ or purely deviatoric loadings $\left(\Sigma_{\mathrm{m}}=0\right)$.

In conclusion, it was demonstrated that for porous solids with homogeneous von Mises matrix and spherical voids, the dilatational response displays a very specific dependence on the signs of the mean stress and third invariant. Although the effect of $\mathrm{J}_{3}^{\Sigma}$ is small, it affects not only the onset of plastic flow of the porous solid, but also void evolution. Small changes in the direction of the normal to the yield surface affects plastic flow of the aggregate and lead to changes in the rate of the damage growth.

It is worth noting that while the analysis was performed for axisymmetric loadings and Rice and Tracey [24] trial velocity field, the same symmetry properties of the dilatational response of porous aggregates hold true for general loadings.

Indeed, for a von Mises matrix, the exact overall potential:

$$
\Pi(\mathbf{D})=\inf _{\mathbf{v} \in K(\mathbf{D})}\langle\pi(\mathbf{d}(\mathrm{v}))\rangle_{\Omega}
$$

is an even function of the macroscopic strain rate tensor D. In Eq. (14), $\Omega$ is the representative volume element, $K(\mathbf{D})$ is the set of incompressible velocity fields compatible with uniform strain rate boundary conditions while \langle\rangle denotes the average value over the representative volume $\Omega$. It follows that the exact macroscopic plastic potential

$$
\mathrm{F}(\boldsymbol{\Sigma})=\sup _{\mathbf{D}}[\boldsymbol{\Sigma}: \mathbf{D}-(\mathbf{D})]
$$

is also even in stresses since:

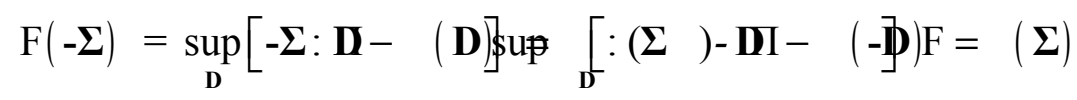


In the above equations ":" denotes the double-contracted product of the two tensors. Since the voids are spherical and randomly distributed in the von Mises matrix, the exact yield function of the porous solids is isotropic, so it depends on the stress tensor only through the isotropic invariants, i.e.

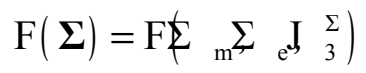

Since $F(\boldsymbol{\Sigma})$ is an even function, it follows that

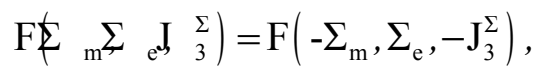

which means that the exact yield surface have the same symmetry properties as the approximate yield criterion developed (see Eq. (12-13) and Fig. 5), namely:

$$
\left\{\begin{array}{l}
\left.\frac{\Sigma_{m}}{\sigma_{T}}\right|_{3} ^{\Sigma}>0, \Sigma_{m} \leq 0=-\left.\frac{\Sigma_{m}}{\sigma_{T}}\right|_{3} ^{\Sigma}<0, \Sigma_{m} \geq 0 \\
\left.\frac{\Sigma_{e}}{\sigma_{T}}\right|_{J_{3}^{\Sigma}>0, \Sigma_{m} \leq 0}=\left.\frac{\Sigma_{e}}{\sigma_{T}}\right|_{3} ^{\Sigma}<0, \Sigma_{m} \geq 0
\end{array}\right.
$$

and that for $\mathrm{J}_{3}^{\Sigma}=0$ the exact yield criterion is symmetric with respect to axis $\Sigma_{\mathrm{m}}=0$.

\section{Comparison with periodic unit cell calculations}

Lebensohn et al. [23] recently extended a full-field spectral formulation based on Fast Fourier Transforms (FFT) [16,17,20] to study the influence of different microstructural features and type of loading on the dilatational viscoplastic behavior of periodic voided materials (porous polycrystalline and homogenous matrix materials). Full details of the 
formulation are given in Lebensohn et al. [23] and will not be repeated. Only some essential aspects of the model implementation for porous materials with homogeneous isotropic matrix are recalled here. A FFT-based calculation requires a discrete description of a periodic unit cell by means of a regularly-spaced grid, which was generated as follows. A cubic domain was partitioned into a regular grid of 128x128x128 Fourier points. To generate a porous material, the Fourier points were randomly picked to try to accommodate (accounting for periodicity) a cavity of radius $\mathrm{r}_{1}$ centered in that point, surrounded by a "security" zone of radius $r_{1}+r_{2}$, where no other cavity was allowed. This random process continued until the target porosity was reached. Setting $r_{1}=6$ and $r_{2}=6.35$ (in units of distance between adjacent Fourier points), a 5\% porosity unit cell with and 109 voids was obtained. Note that a unit cell generated in this way is only an approximation of a strictly isotropic porous material. To mitigate this concern, we have verified that the flow stresses corresponding to axisymmetric loadings along the three orthogonal directions that define the cubic domain are almost identical.

All Fourier points not belonging to the cavities were assigned a material constitutive behavior $\mathbf{d}(\mathrm{x})=\partial \varphi(\mathrm{x}, \sigma) / \partial \sigma$, deriving from an incompressible, isotropic power-law potential of the form (see Duva and Hutchinson [6]):

$\varphi(\mathrm{x}, \sigma)=\frac{\sigma_{\mathrm{o}} \gamma_{\mathrm{o}}}{\mathrm{n}+1}\left(\frac{\sigma_{\mathrm{e}}(\mathrm{x})}{\sigma_{\mathrm{o}}}\right)^{\mathrm{n}+1}$,

where $\sigma_{\mathrm{e}}(\mathbf{x})$ is the local von Mises equivalent stress, $\sigma_{\mathrm{o}}$ and $\gamma_{\mathrm{o}}$ are reference stress and strain rate, respectively, and $n$ is the stress exponent. The stress potential inside a void is $\varphi(\mathrm{x}, \sigma)=0$ if $\boldsymbol{\sigma}=0$, and infinity otherwise. The effective viscoplastic behavior of the aggregate is defined as the relation between the average stress $\Sigma=\langle\boldsymbol{\sigma}(\mathbf{x})\rangle$ and the average strain rate $\mathbf{D}=<\mathbf{d}(\mathbf{x})>$ over the aggregate. Formally, it is given by: 
$\mathrm{D}=\frac{\partial \Phi}{\partial \Sigma}(\Sigma) ; \quad \Phi(\Sigma)=\min _{\sigma \in \mathrm{S}(\Sigma)}\langle\varphi(\mathrm{x}, \sigma)\rangle$

(21)

where $\Phi(\Sigma)$ is the overall stress potential for the porous aggregate and $\mathbf{S}(\boldsymbol{\Sigma})$ denotes the set of statically-admissible stress fields with prescribed average $\boldsymbol{\Sigma}$. The local and effective potentials are homogeneous functions of degree $(n+1)$ in $\boldsymbol{\sigma}$ and $\boldsymbol{\Sigma}$, respectively (see Duva and Hutchinson [6], Ponte Castaneda and Suquet [12]). Therefore, the equipotential surface $\Phi(\Sigma)=$ constant, fully characterizes the dilatational response (see also Leblond et al [29]). Results are reported here in the form of equipotential (or "gauge") surfaces given by: 


$$
\left\{\Sigma^{*}: \Phi\left(\Sigma^{*}\right)=\frac{\sigma_{\mathrm{o}}^{-\mathrm{n}} \gamma_{\mathrm{o}}}{\mathrm{n}+1}\right\}
$$

A more convenient equation can be obtained by writing the effective potential as:

$$
\Phi(\Sigma)=\frac{\sigma_{\mathrm{o}} \gamma_{\mathrm{o}}}{\mathrm{n}+1}\left(\frac{\Lambda(\Sigma)}{\sigma_{\mathrm{o}}}\right)^{\mathrm{n}+1}
$$

where the so-called gauge factor $\Lambda(\boldsymbol{\Sigma})$ is a homogeneous function of degree 1 in $\boldsymbol{\Sigma}$ and the $\boldsymbol{\Sigma}^{*}=\boldsymbol{\Sigma} / \Lambda(\boldsymbol{\Sigma})$ lies on the gauge surface. Points on the gauge surface can be determined by computing the effective stress potential for an applied macroscopic stress of arbitrary magnitude, and rescaling the latter accordingly. In the limit when the power law exponent tends to infinity, the theory also applies to rate-independent perfect plasticity with von Mises yield criterion (see Duva and Hutchinson [6], Leblond et al. [29]). Figure 6 shows the yield surfaces according to the new analytic yield criterion and Gurson's [1] criterion and the gauge surfaces obtained numerically for a stress exponent $\mathrm{n}$ $=20$. The gauge surface of a porous material with power-law matrix and $n=20$ viscoplastic behaviour will differ slightly from the yield surface of an analogous rateinsensitive material, the exact difference depending on porosity and triaxiality. Yet, the main observation is that the unusual features of the dilatational response predicted by the new criterion, namely the sensitivity to the sign of loading for triaxialities different from 0 and $\pm \infty$ and the very specific coupling between the mean stress and third invariant predicted by the new criterion are indeed confirmed by the full-field results. The dilatational response predicted by the FFT-based method is softer than that predicted by the new analytical criterion. This softer behavior predicted by the full-field calculation is to be expected since in this approach distributed porosity was considered, which is known to imply stronger void interaction, and therefore a slightly softer effective response, compared with the hollow sphere solution Ponte Castaneda et Suquet [12]. 


\section{Summary and Conclusions}

In this paper, we demonstrated that the insensitivity of Gurson's criterion to the third invariant and the ensuing strong symmetry properties of the yield surface are direct consequences of a simplifying hypothesis, namely that the "crossed term" $\mathrm{D}_{m} \mathrm{D}_{11}^{\prime}$ in the expression of the plastic strain rate dissipation can be neglected. Specifically, it was shown that neglecting this term amounts to neglecting the third invariant influence as well as coupling effects between the mean stress and the stress deviator on yielding of the porous aggregate. Furthermore, a new yield criterion was developed in closed form (the parametric representation of the yield surface is given by Eqs. (12-13)). This criterion predicts that for stress-triaxialities different from 0 and $\pm \infty$, the dilatational response is sensitive to the signs of the mean stress and the third invariant. Gurson criterion is an upper-bound for the new criterion, only for purely hydrostatic or purely deviatoric states, the new criterion coincides with Gurson's. Specifically, while Gurson (1977) is invariant to both transformations $\left(\Sigma_{\mathrm{m}}, \Sigma^{\prime}\right) \rightarrow\left(\Sigma_{\mathrm{m}},-\Sigma^{\prime}\right)$ and $\left.\left(\Sigma_{\mathrm{m}}, \Sigma^{\prime}\right) \rightarrow\left(-\Sigma_{\mathrm{m}}, \Sigma^{\prime}\right)\right)$, the new criterion is invariant only to $\left(\Sigma_{\mathrm{m}}, \Sigma^{\prime}\right) \rightarrow\left(-\Sigma_{\mathrm{m}},-\Sigma^{\prime}\right)$, and is insensitive to the sign of $\Sigma_{\mathrm{m}}$ only for purely deviatoric states $\left(\Sigma_{\mathrm{m}}=0\right)$. Also, according to the proposed criterion for $\Sigma_{\mathrm{m}} \geq 0$, the response is softer for $\mathrm{J}_{3}^{\Sigma} \geq 0$ than for $\mathrm{J}_{3}^{\Sigma} \leq 0$ (see Fig. 4). For purely deviatoric loading, the response is insensitive to the sign of $\mathrm{J}_{3}^{\Sigma}$ while there is a slight $\mathrm{J}_{3}^{\Sigma}$ effect with increasing triaxiality (see Figs. 4a-b). The normal to the yield surface corresponding to $\mathrm{J}_{3}^{\Sigma} \geq 0$ and $\mathrm{J}_{3}^{\Sigma} \leq 0$, respectively, are clearly different, determining distinct plastic flow directions and resulting void growth rates. For triaxialities approaching infinity, the effect of $\mathrm{J}_{3}^{\Sigma}$ starts to decrease and as expected it is nil for purely hydrostatic loading (see Fig. 4c). 
In summary, although the effect of the third invariant on yielding is small, its effects on void evolution may become large, considering that very small changes in the direction of the normal to the yield surface affect plastic flow of the aggregate and may lead to large changes in the rate of the damage growth. A full-field dilatational viscoplastic FFT-based approach was also used to generate gauge surfaces for periodic viscoplastic porous materials with matrix's response depending only on the von Mises effective stress and subject to axisymmetric loading conditions. The numerical calculations reveal the same new trends as the approximate yield criterion (Eq. 12-13), namely: a very specific coupling between the third invariant and mean stress. Furthermore, it was also shown that the exact criterion for a porous solid with von Mises matrix has the same unusual properties. This coupling and the resulting lack of symmetry of the yield surface of porous solids with isotropic matrix described by a plastic potential even in stresses deserve further systematic experimental and numerical investigations.

\section{References}

[1] Gurson, A. L., 1977, "Continuum theory of ductile rupture by void nucleation and growth. Part I: Yield criteria and flow rules for porous ductile media". ASME J. Eng. Mater., Series H, 99, pp 2-15.

[2] Tvergaard, V., 1981, "Influence of voids on shear band instabilities under plane strain conditions", Int. J. Fracture, 17, pp 389-407.

[3] Tvergaard, V. and Needleman, A.,1984, "Analysis of the cup-cone fracture in a round tensile bar", Acta Metall, 32 (1), pp 157-69.

[4] Monchiet, V., Charkaluk, E., and Kondo, D., 2011, "A micromechanics-based modification of the Gurson criterion by using Eshelby-like velocity fields", European Journal of Mechanics - A/Solids, 30, pp 940-949.

[5] Leblond, J.-B, 2003, Mécanique de la rupture fragile et ductile. Ed. Hermes.

[6] Duva, J.M., Hutchinson, J.W, 1984, " Constitutive potentials for dilutely voided nonlinear materials", Mech. Mater., 3, pp 41-54.

[7] Ponte Castañeda, P., 1991, "The effective mechanical properties of nonlinear isotropic materials", J. Mech. Phys. Solids, 39, pp 45-71.

[8] Kaisalam, M., Ponte Castañeda, P., 1998, "A general constitutive theory for linear and nonlinear particulate media with microstructure evolution", J. Mech. Phys. Solids, 46, pp 427-465. 
[9] Richelsen, A.B., Tvergaard, V., 1994, "Dilatant plasticity or upper bound estimates for porous ductile solids", Acta Metall. Mater., 42, pp 2561-2577.

[10] Kim, J., Gao, X., Srivatsan, T.S., 2004, "Modeling of void growth in ductile solids: effects of stress triaxiality and initial porosity", Eng. Fract. Mech, 71, pp 379400.

[11] McElwain, D.L.S., Roberts, A.P., Wilkins, A.H., 2006, "Yield criterion of porous materials subjected to complex stress states", Acta Mater. ,54, pp 1995-2002.

[12] Ponte Castañeda, P., Suquet, P., 1998, "Nonlinear composites", Adv. Appl. Mech., 34, pp 171-302.

[13] Danas, K., Idiart, M.I. Ponte Castañeda, P. ,2008, "A homogenization-based constitutive model for isotropic viscoplastic porous media", Int. J. Solids Struct., 45, pp 3392-3409.

[14] Ponte Castañeda, P., 2002, "Second-order homogenization estimates for nonlinear composites incorporating field fluctuations: I- Theory", J. Mech. Phys. Solids, 50, pp 737-757.

[15] Julien, J., Garajeu, M. and Michel, J-C., 2011. "A semi-analytical model for the behavior of saturated viscoplastic materials containing two populations of voids of different sizes" Int. J. Solids. Structures, 48, pp. 1485-1498.

[16] Moulinec, H., Suquet, P., 1998, "A numerical method for computing the overall response of nonlinear composites with complex microstructure", Comput. Methods Appl. Mech. Engrg., 157, pp 69-94.

[17] Michel, J.C., Moulinec, H., Suquet, P., 2000, "A computational method based on augmented Lagrangians and fast Fourier transforms for composites with high contrast", Comp. Mod. Eng. Sci., 1, pp 79-88.

[18] Stewart, J. B., Cazacu, O., 2009, "Plastic potentials for porous aggregates with the matrix exhibiting tension-compression asymmetry", Journal of Mechanics and Physics of Solids, 57, pp 325-341.

[19] Lebensohn, R.A., Cazacu, O., 2012, "Effect of single-crystal plastic deformation mechanisms on the dilatational plastic response of porous polycrystals", Int. J. Solids Struct., accepted. 
[20] Lebensohn, R.A., 2001, "N-site modeling of a 3-D viscoplastic polycrystal using fast Fourier transform", Acta Mater., 49, pp 2723-2737.

[21] Bao, Y., Wierzbicki, T., 2004. "On fracture locus in the equivalent strain and stress triaxiality space", Int. J. Mech. Sci., 46, pp 81-98.

[22] Cazacu, O., Plunkett, B., Barlat, F., 2006, "Orthotropic yield criterion for hexagonal closed packed materials", International Journal of Plasticity, 22, pp 11711194.

[23] Lebensohn, R.A., Idiart, M.I., Ponte Castaneda, P., Vincent, P.-G., 2011, "Dilatational viscoplasticity of polycrystalline solids with intergranular cavities", Phil. Mag., 91, pp 3038-3067.

[24] Rice, J.R., Tracey, D.M., 1969, "On the ductile enlargement of voids in triaxial stress fields", J. Mech. Phys. Solids, 17, pp. 201-217.

[25] Hill, R., 1967, "The essential structure of constitutive laws of metal composites and polycrystals". J. Mech. Phys. Solids, 15, pp. 79-95.

[26] Mandel, J., "Plasticité classique et viscoplasticité", Int. Centre Mech Sci., Courses and lectures, 97, Udine 1971, Springer, Wien, New York 1972.

[27] Gurson, A, "Plastic flow and fracture behavior of ductile materials incorporating void nucleation, growth, and interaction. 1975, Ph.D. thesis, Brown University.

[28] Gărăjeu, M., 2011. Comportement non linéaire des milieux hétérogènes. Habilitation (HDR) Thesis, Université Paul Cézanne, Marseille, France (in French).

[29] Leblond, J.-B., Perrin, G., Suquet, P., 1994, "Exact results and approximate models for porous viscoplastic solids", Int. J. Plasticity 10, 213-225. 


\section{Appendix: Parametric representation of the analytic yield surface}

A1) For $\mathrm{D}_{11}^{\prime}=\frac{\mathrm{D}_{\mathrm{e}}}{2}>0$ and $\mathrm{D}_{\mathrm{m}}=\frac{1}{3} \operatorname{tr} \mathbf{D} \geq 0$ :

It follows that $u=2 \frac{\left|D_{m}\right|}{D_{e}}=2 \frac{D_{m}}{D_{e}}$, and the overall plastic potential of the porous solid is given by (see Eq. (10)):

$\Pi^{+}(\mathbf{D})=\frac{\sigma_{\mathrm{T}}}{\mathrm{V}} \int_{\Omega} \sqrt{4 \mathrm{D}_{\mathrm{m}}^{2}\left(\frac{\mathrm{b})^{6}}{\mathrm{r}^{\circ}}+\mathrm{D}_{\mathrm{e}}^{2}+\mathrm{D}_{\mathrm{e}}\left|\mathrm{D}_{\mathrm{p}}^{(}\right|{\frac{\mathrm{b}^{3}}{\dot{\overrightarrow{\mathrm{r}}}}}^{3}(1+3 \cos 2 \theta)\right.} \mathrm{d} \Omega$

with $V=4 \pi b^{3} / 3$ and $d V=r^{2} \sin \theta d \theta d \varphi d r$. Using the change of variable, $y=\frac{u b^{3}}{r^{3}}$ and $\alpha=\cos \theta$, respectively, the integral (A.1) can be written in the form:

$\Pi^{+}(\mathbf{D})=\sigma_{\mathrm{T}} \mathrm{D}_{\mathrm{e}} u \int_{u}^{u / f} \int_{0}^{1} \frac{\sqrt{1+\mathrm{y}^{2}+\mathrm{y}\left(3 \alpha^{2}-1\right)}}{\mathrm{y}^{2}} \mathrm{~d} \alpha \mathrm{dy}$

Note that:

$$
\int_{0}^{1} \frac{\sqrt{1+\mathrm{y}^{2}+\mathrm{y}\left(3 \alpha^{2}-1\right)}}{\mathrm{y}^{2}} \mathrm{~d} \alpha=\frac{1+y}{2 y^{2}}+\frac{1+y^{2}-y}{2 \sqrt{3} y^{5 / 2}} \ln \left(\frac{1+y+\sqrt{3 y}}{\sqrt{1+y^{2}-y}} \dot{\overline{\mathrm{j}}}\right.
$$

Further integration with respect to $y$ leads to:

$\Pi^{+}(\mathbf{D})=\sigma_{\mathrm{T}} \mathrm{D}_{\mathrm{e}} \mathrm{u}[\mathrm{F}(\sqrt{\mathrm{u} / \mathrm{f}})-\mathrm{F}(\sqrt{\mathrm{u}})]$

with: 


$$
\begin{aligned}
F(z) & =-\frac{2}{3 z^{2}}+\frac{1}{3 \sqrt{3}}[\arctan (2 z+\sqrt{3})-\arctan (2 z-\sqrt{3})] \\
& +\frac{1}{2} \ln \left(z^{4}-z^{2}+1\right)+\ln \left(\frac{\left.z^{2}+z \sqrt{3}+1\right)}{z^{2}-z \sqrt{3}+1 \frac{\dot{*}}{\dot{*}}} \frac{3 z^{4}+3 z^{2}-1}{6 \sqrt{3} z^{3}}\right.
\end{aligned}
$$

It follows that the parametric representation of the yield surface is:

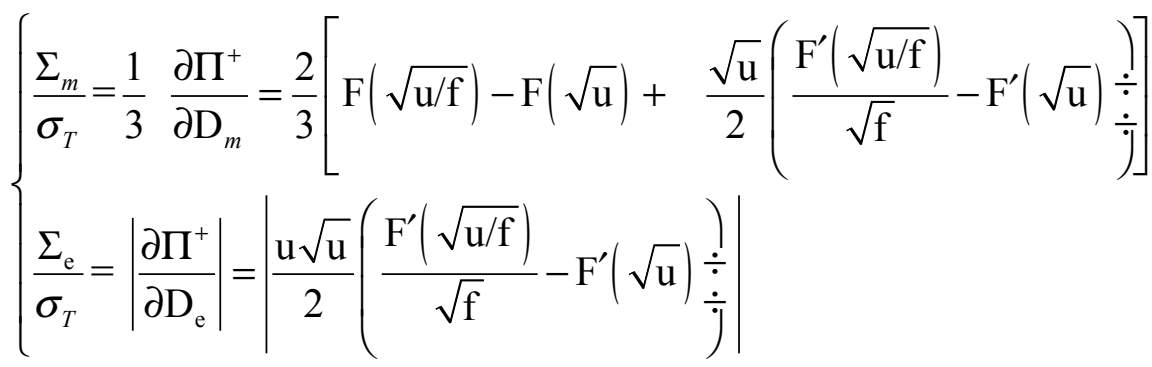

where the prime symbol indicates first derivative. Substitution of (A.5) into (A.6) leads to Eq. (12).

A2) For $\mathrm{D}_{11}^{\prime}=\frac{\mathrm{D}_{\mathrm{e}}}{2}>0$ and $\mathrm{D}_{\mathrm{m}}=\frac{1}{3} \operatorname{tr} \mathbf{D} \leq 0$ :

It follows that $u=2 \frac{\left|D_{m}\right|}{D_{e}}=-2 \frac{D_{m}}{D_{e}}$, and the overall plastic potential of the porous solid (see Eq. (11)) is:

$\Pi^{+}(\mathbf{D})=\sigma_{\mathrm{T}} \mathrm{D}_{\mathrm{e}} u \int_{u}^{u / f} \int_{0}^{1} \frac{\sqrt{1+\mathrm{y}^{2}-\mathrm{y}\left(3 \alpha^{2}-1\right)}}{\mathrm{y}^{2}} \mathrm{~d} \alpha \mathrm{dy}$

Note that:

$\int_{0}^{1} \frac{\sqrt{1+\mathrm{y}^{2}-\mathrm{y}\left(3 \alpha^{2}-1\right)}}{\mathrm{y}^{2}} \mathrm{~d} \alpha=\frac{|y-1|}{2 y^{2}}+\frac{1+y^{2}+y}{2 \sqrt{3} y^{5 / 2}} \tan ^{-1}\left(\frac{\sqrt{3 y})}{|y-1| \frac{\dot{\hat{1}}}{0}}\right.$

Further integration with respect to $y$ leads to: 


$$
\left\{\begin{array}{l}
\Pi^{+}(\mathbf{D})=\sigma_{\mathrm{T}} \mathrm{D}_{\mathrm{e}} \mathrm{u}[\mathrm{G}(\sqrt{\mathrm{u} / \mathrm{f}})-G(\sqrt{\mathrm{u}})], \forall u<f \\
\Pi^{+}(\mathbf{D})=\sigma_{\mathrm{T}} \mathrm{D}_{\mathrm{e}} \mathrm{u}\left[\mathrm{G}(\sqrt{\mathrm{u}})+G(\sqrt{\mathrm{u} / \mathrm{f}})+2 \ln (3)-\frac{2}{9} \frac{\pi]}{\sqrt{3}]} \mid, \forall f<u<1\right. \\
\Pi^{+}(\mathbf{D})=\sigma_{\mathrm{T}} \mathrm{D}_{\mathrm{e}} \mathrm{u}[\mathrm{G}(\sqrt{\mathrm{u}})-G(\sqrt{\mathrm{u} / \mathrm{f}})], \forall u>1
\end{array}\right.
$$

with:

$$
\begin{aligned}
& G(z)=\frac{3 z^{4}-3 z^{2}-1}{3 \sqrt{3} z^{3}} \tan ^{-1}\left(\frac{z \sqrt{3}}{1-z^{2 \cdot \frac{2}{9}}}+\frac{1}{3 \sqrt{3}}\left(\tan ^{-1}\left(\frac{2 z+1}{\sqrt{3}}\right)-\tan ^{-1} \mid \frac{2 z-1}{\sqrt{3})^{\div}} \div\right)\right. \\
& -\frac{1}{2} \ln \left(z^{4}+z^{2}+1\right)-\frac{2}{3 z^{2}}
\end{aligned}
$$




\section{Figure Captions}

Figure 1: Yield surface according to the new criterion, corresponding to $\mathrm{J}_{3}^{\Sigma} \leq 0$ and porosity $\mathrm{f}=0.05$, showing sensitivity of yielding to the sign of the mean stress.

Figure 2: Yield surface according to the new criterion, corresponding to $\mathrm{J}_{3}^{\Sigma} \geq 0$ for porosity $\mathrm{f}=0.05$.

Figure 3: Yield surface of the porous solid according to proposed criterion for $\mathrm{J}_{3}^{\Sigma} \leq 0$ and $\mathrm{J}_{3}^{\Sigma} \geq 0$, respectively, and comparison with Gurson (1977) for porosity $\mathrm{f}=0.05$.

Figure 4: Zoom on the tensile quadrant of the yield surfaces according to the new yield criterion, for $\mathrm{J}_{3}^{\Sigma} \leq 0$ and $\mathrm{J}_{3}^{\Sigma} \geq 0$, and Gurson (1977) criterion, for porosity $\mathrm{f}=0.05$, within following ranges: (a) $\left(\Sigma_{\mathrm{m}} \geq 0,0.8<\Sigma_{\mathrm{e}}<(1-\mathrm{f})\right)$; (b) $\left(\Sigma_{\mathrm{m}} \geq 0,0.3<\Sigma_{\mathrm{e}}<0.8\right)$ : (c) $\left(\Sigma_{\mathrm{m}} \geq 0\right.$, $\left.0<\Sigma_{\mathrm{e}}<0.5\right)$.

Figure 5: Yield surfaces obtained with the new yield criterion, and with Gurson (1977), for porosities $\mathrm{f}=1 \%, 5 \%$ and $15 \%$. The blue lines represent the yield surfaces for $\mathrm{J}_{3}^{\Sigma} \leq 0$ and the red lines for $\mathrm{J}_{3}^{\Sigma} \geq 0$. The dashed lines are Gurson's yield surface.

Figure 6: Yield surfaces obtained with the new yield criterion (solid lines) and with Gurson (1977) (dashed line), and points belonging to gauge surfaces calculated with the FFT-based model for a periodic until cell. In all cases, porosity $f=0.05$. The blue line and symbol correspond to $\mathrm{J}_{3}^{\Sigma} \leq 0$, and the red line and symbol to $\mathrm{J}_{3}^{\Sigma} \geq 0$. 


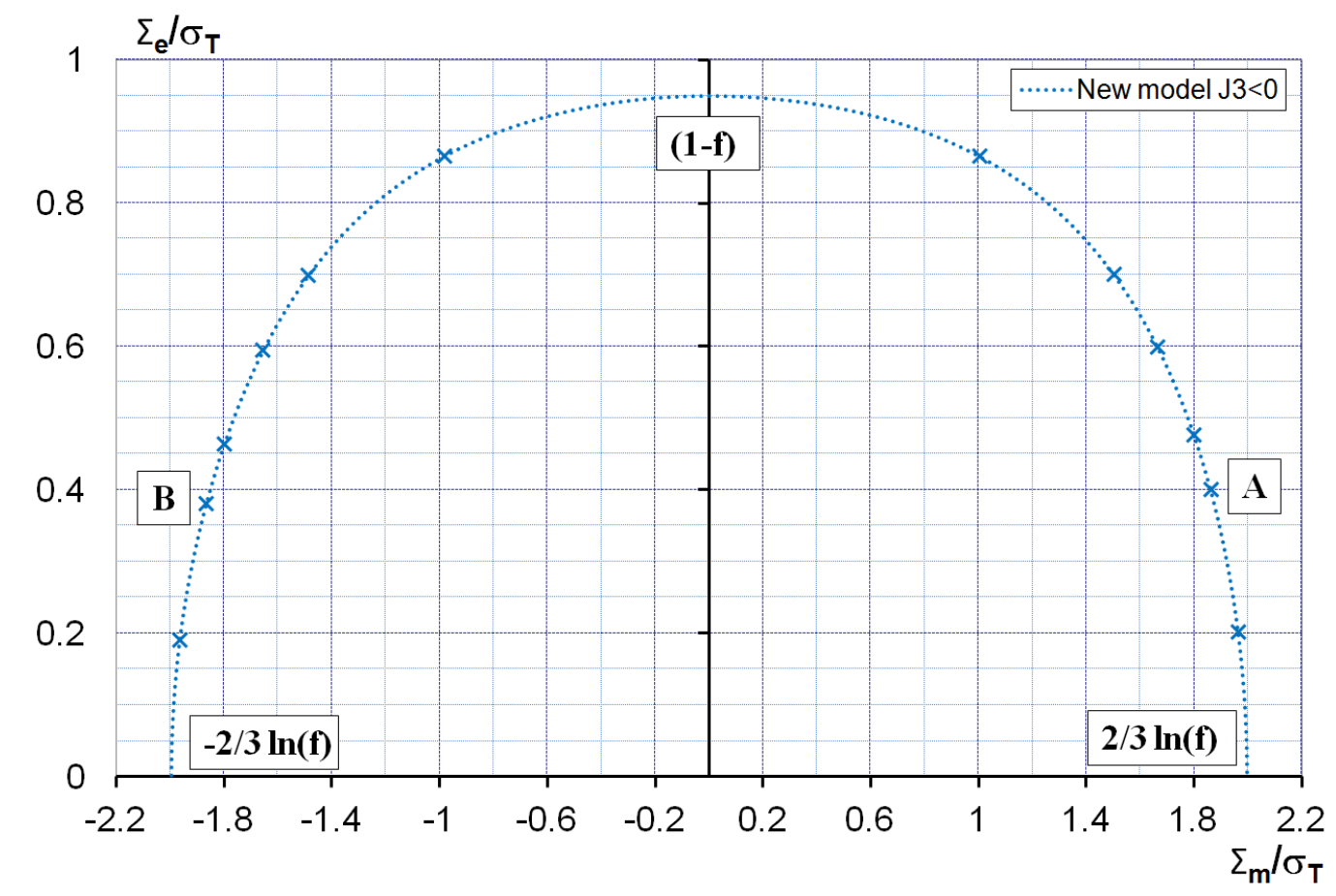

Figure 1: Yield surface according to the new criterion, corresponding to $\mathrm{J}_{3}^{\Sigma} \leq 0$ and porosity $\mathrm{f}=0.05$, showing sensitivity of yielding to the sign of the mean stress. 


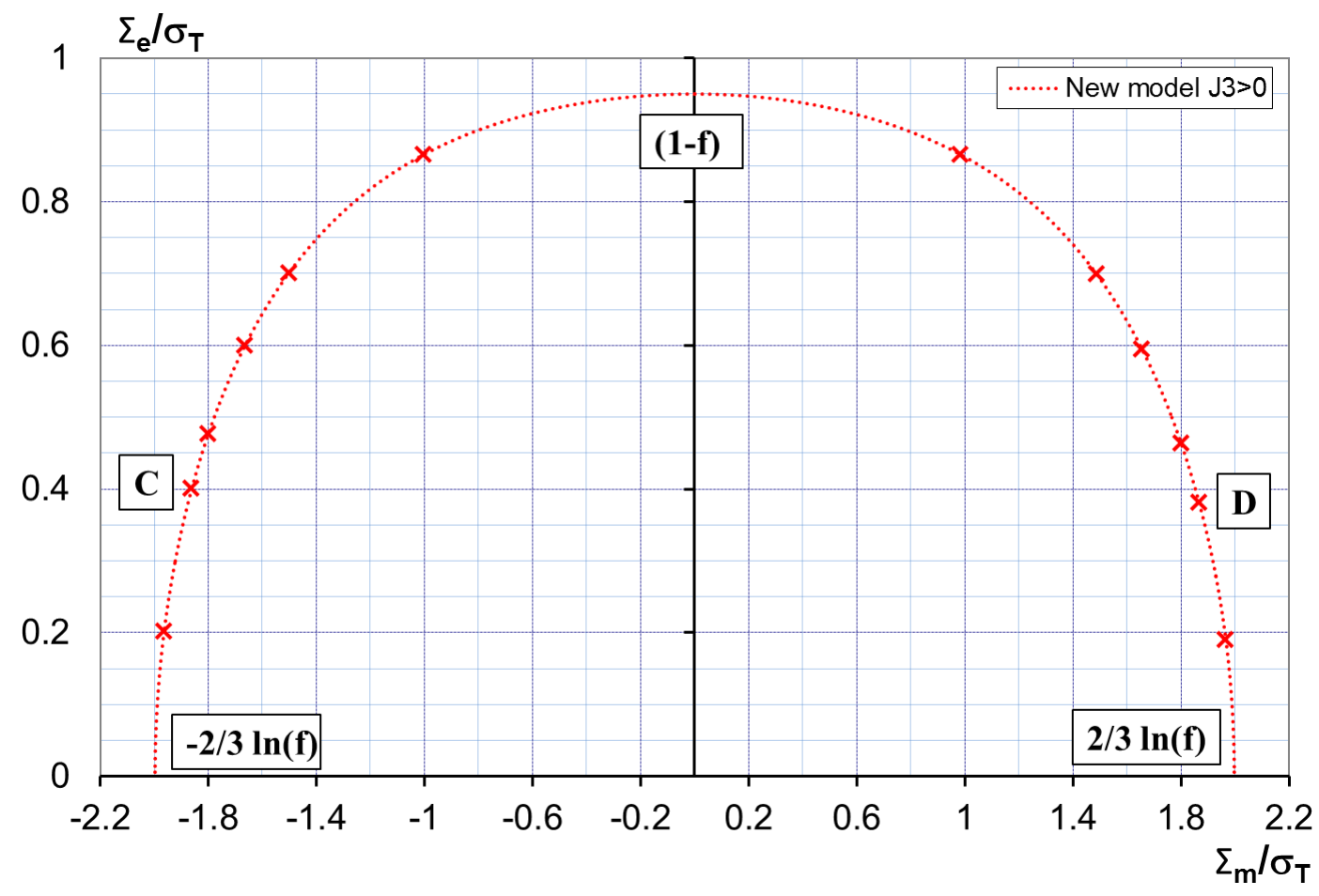

Figure 2: Yield surface according to the new criterion, corresponding to $\mathrm{J}_{3}^{\Sigma} \geq 0$ and porosity $\mathrm{f}=0.05$. 


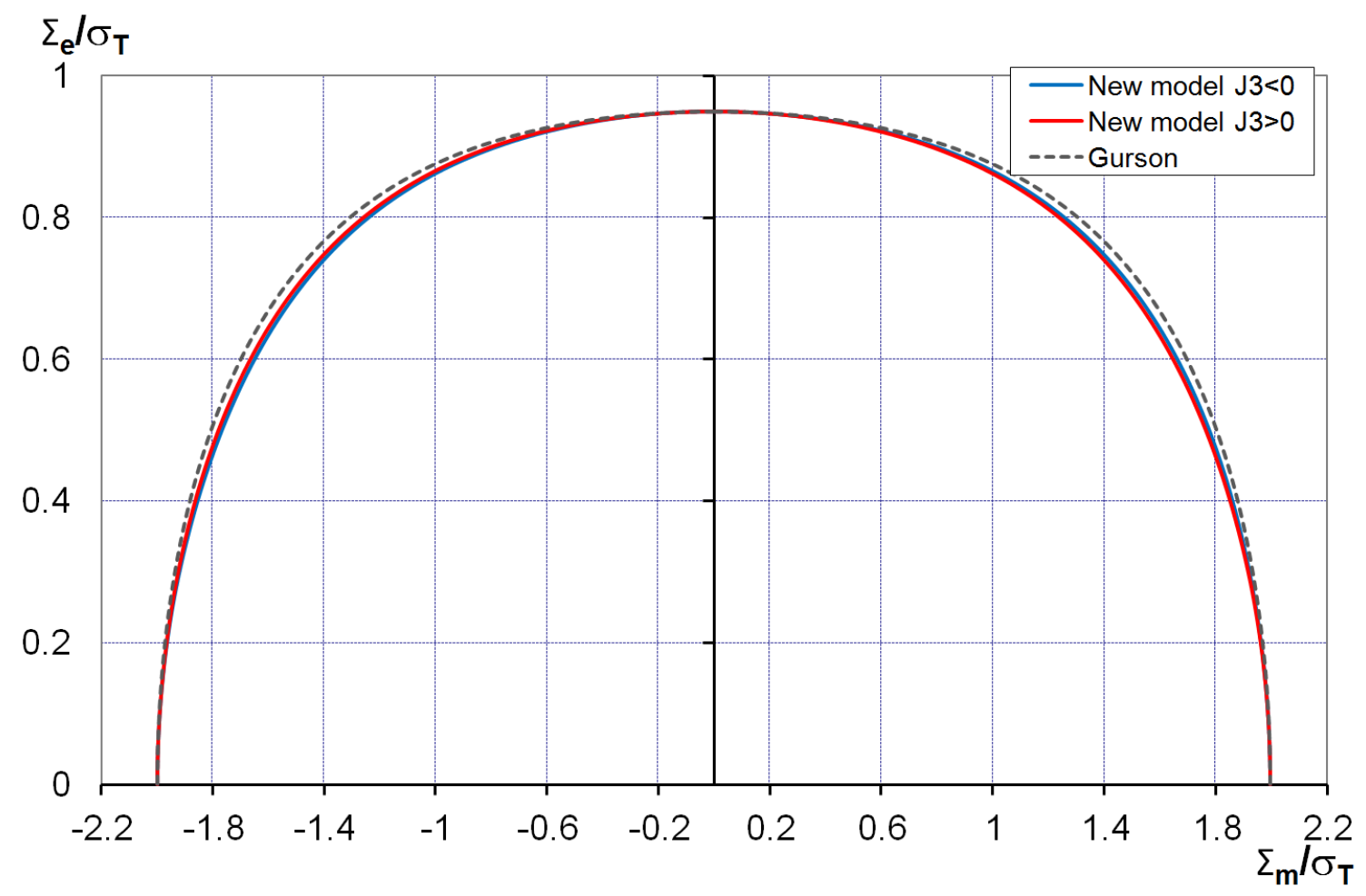

Figure 3: Yield surface of the porous solid according to proposed criterion for $\mathrm{J}_{3}^{\Sigma} \leq 0$ and $J_{3}^{\Sigma} \geq 0$, respectively, and comparison with Gurson (1977) for porosity $f=0.05$. 


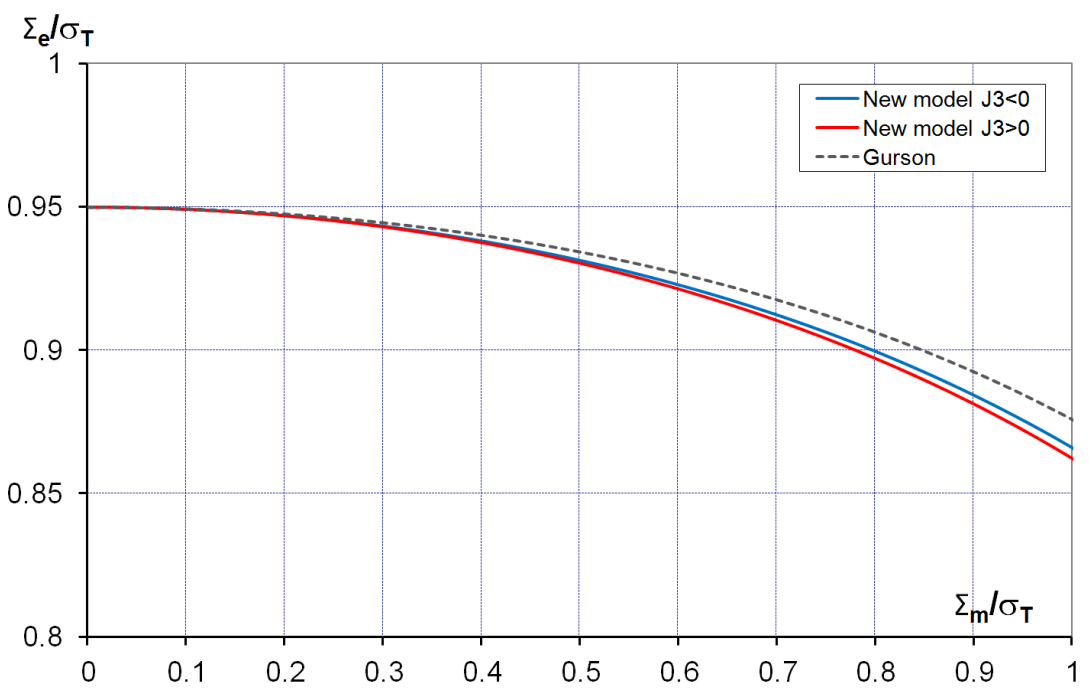

(a)

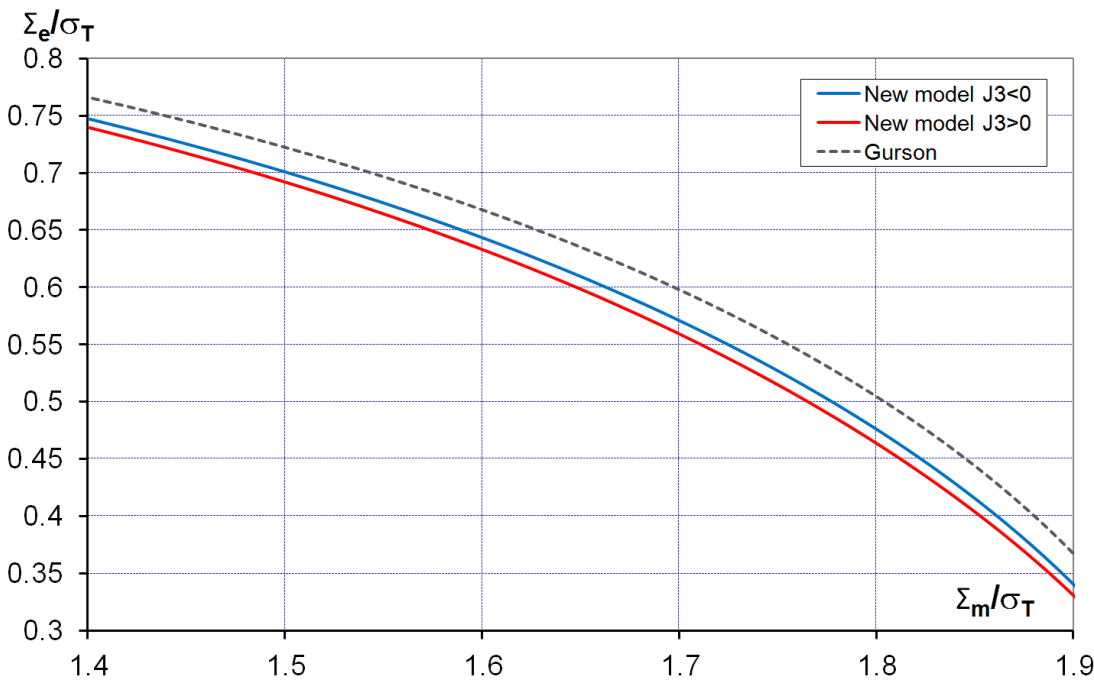

(b)

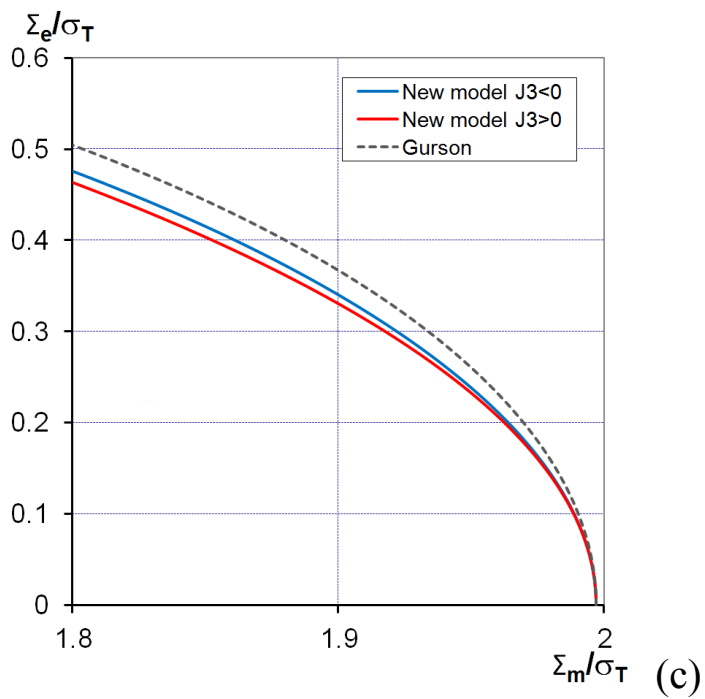


Figure 4: Zoom on the tensile quadrant of the yield surfaces according to the new yield criterion, for $\mathrm{J}_{3}^{\Sigma} \leq 0$ and $\mathrm{J}_{3}^{\Sigma} \geq 0$, and Gurson (1977) criterion, for porosity $\mathrm{f}=0.05$, within following ranges: (a) $\left(\Sigma_{\mathrm{m}} \geq 0,0.8<\Sigma_{\mathrm{e}}<(1-\mathrm{f})\right)$; (b) $\left(\Sigma_{\mathrm{m}} \geq 0,0.3<\Sigma_{\mathrm{e}}<0.8\right)$ : (c) $\left(\Sigma_{\mathrm{m}} \geq 0\right.$, $\left.0<\Sigma_{\mathrm{e}}<0.5\right)$. 


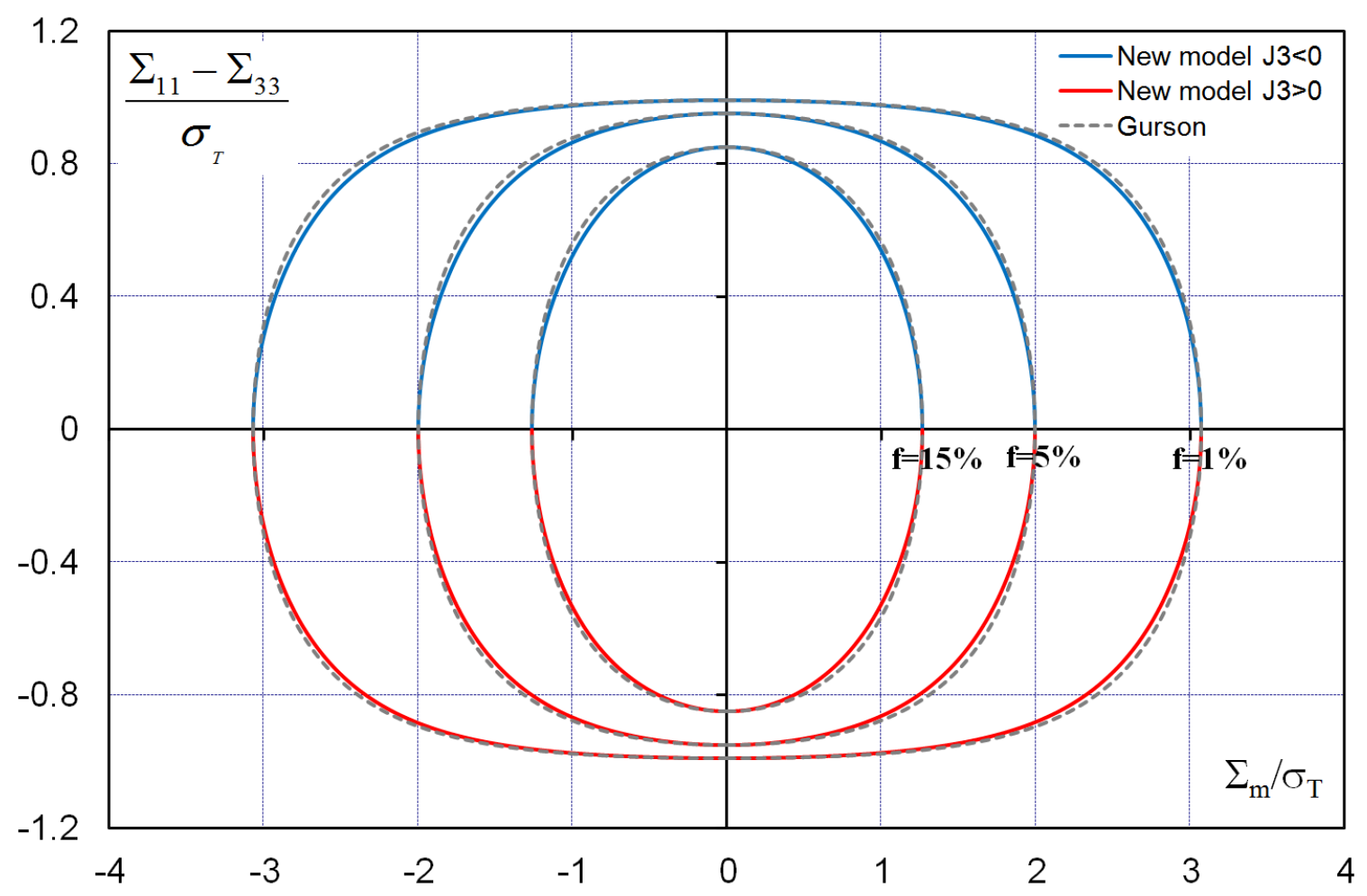

Figure 5: Yield surfaces obtained with the new yield criterion, and with Gurson (1977), for porosities $\mathrm{f}=1 \%, 5 \%$ and $15 \%$. The blue lines represent the yield surfaces for $\mathrm{J}_{3}^{\Sigma} \leq 0$ and the red lines for $\mathrm{J}_{3}^{\Sigma} \geq 0$. The dashed lines are Gurson's yield surface. 


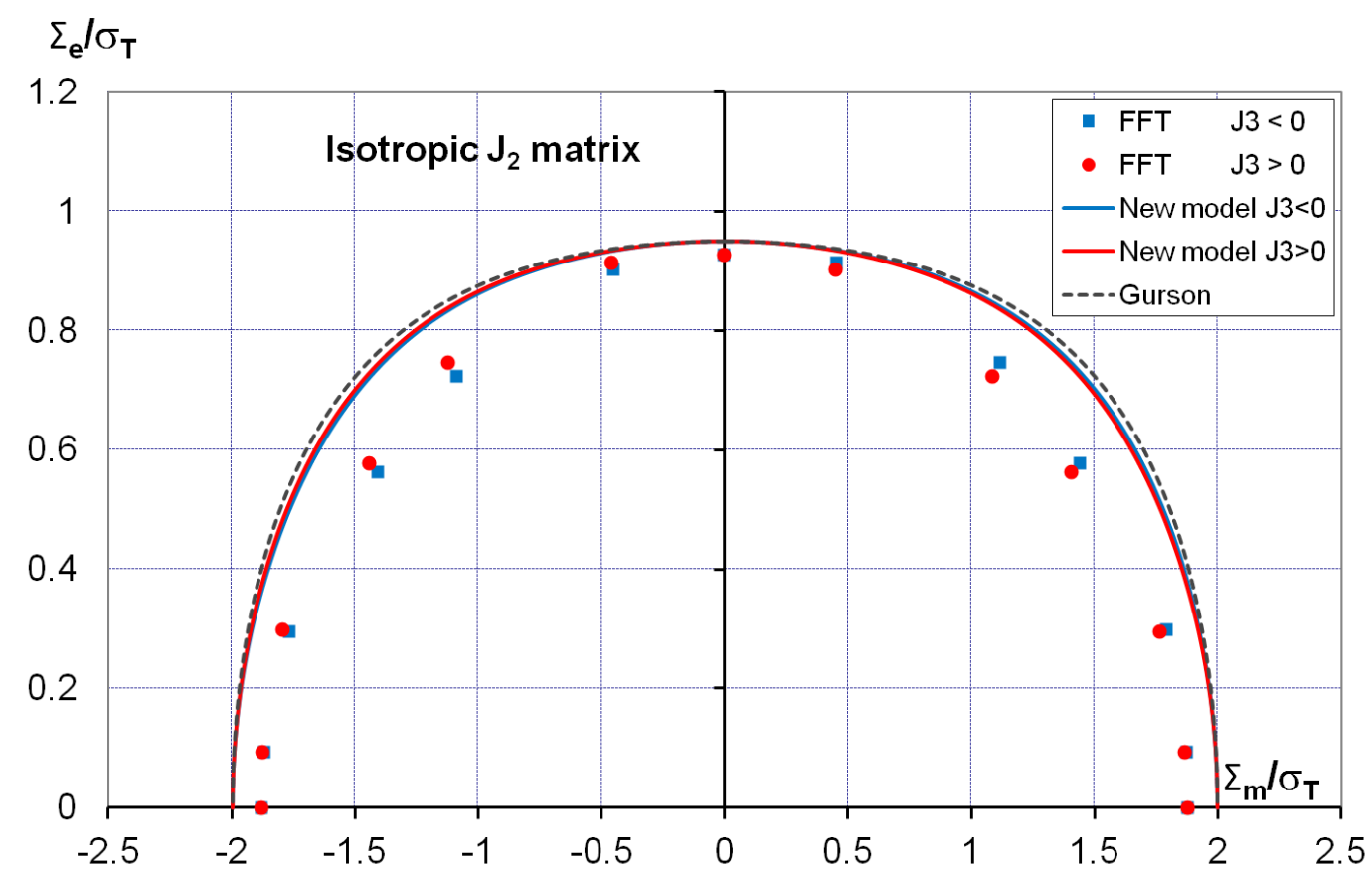

Figure 6: Yield surfaces obtained with the new yield criterion (solid lines) and with Gurson (1977) (dashed line), and points belonging to gauge surfaces calculated with the FFT-based model for a periodic until cell. In all cases, porosity $f=0.05$. The blue line and symbol correspond to $\mathrm{J}_{3}^{\Sigma} \leq 0$, and the red line and symbol to $\mathrm{J}_{3}^{\Sigma} \geq 0$. 